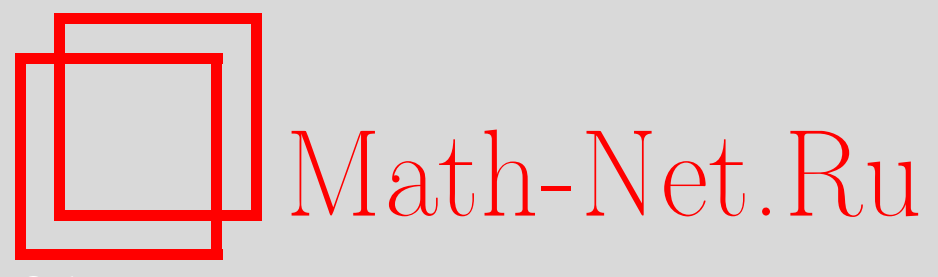

М. С. Лобанов, Точное соотношение между нелинейностью и алгебраической иммунностью, Дискрет. матем., 2006, том 18, выпуск 3, 152-159

DOI: https://doi.org/10.4213/dm67

Использование Общероссийского математического портала Math-Net.Ru подразумевает, что вы прочитали и согласны с пользовательским соглашением http: //www . mathnet.ru/rus/agreement

Параметры загрузки:

IP: 54.162 .27 .143

26 апреля 2023 г., 10:34:01

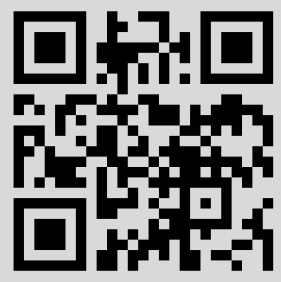




\title{
Точное соотношение между нелинейностью и алгебраической иммунностью
}

\author{
(c) 2006 г. . М. Лобанов
}

\begin{abstract}
Булевы функции нашли широкое применение в криптографии. В связи с появлением "алгебраической” атаки на потоковые шифры, к булевым функциям, используемым в этих шифрах в качестве нелинейных фильтров, стало, наряду с другими, предъявляться требование обладания высокой алгебраической иммунностью. Еще одним из наиболее важных криптографических свойств булевых функций, особенно используемых в потоковых шифрах, является нелинейность. В связи с этим представляет интерес вопрос связи нелинейности булевой функции с ее алгебраической иммунностью.

В настоящей работе мы получим нижнюю оценку нелинейности через значение алгебраической иммунности и построим функции, на которых эта оценка достигается при любых допустимых значениях параметров.
\end{abstract}

Булевы функции нашли широкое применение в криптографии. В последнее время в связи с появлением так называемой “алгебраической” атаки на потоковые шифры (см. например $[1,5])$, к булевым функциям, используемым в этих шифрах в качестве нелинейных фильтров, стало наряду с другими предъявляться требование обладания высокой алгебраической иммунностью. Еще одним из наиболее важных криптографических свойств булевых функций, особенно используемых в потоковых шифрах, является нелинейность. В связи с этим очень интересен вопрос, как связаны нелинейность булевой функции и ее алгебраическая иммунность.

В [2] было доказано утверждение, позволяющее оценивать снизу нелинейность функции через значение ее алгебраической иммунности.

В настоящей работе мы получим более сильную нижнюю оценку нелинейности через значение алгебраической иммунности и построим функции, на которых эта оценка достигается при любых допустимых значениях параметров.

Известно, что булева функщия единственным образом представляется полиномом.

Определение 1. Степенью булевой функщии называется длина самого длинного слагаемого в ее полиноме (количество переменных в этом слагаемом).

Определение 2. Булева функция $g$ над $F_{2}^{n}$ называется аннигилятором булевой функции $f$ над $F_{2}^{n}$, если $f g=0$.

Очевидно, что аннигиляторы $f$ образуют линейное подпространство в пространстве всех булевых функщий от $n$ переменных. 
Определение 3. Алгебраической иммунностью $\mathrm{AI}(F)$ булевой функции $f$ над $F_{2}^{n}$ называется степень булевой функции $g$ над $F_{2}^{n}$, где $g$ не равная тождественно нулю функция с минимальной степенью, такая, что $f g=0$ или $(f+1) g=0$.

Известно $[1,5]$, что для любой $f$ над $F_{2}^{n}$

$$
\mathrm{AI}(f) \leqslant\left\lceil\frac{n}{2}\right\rceil \text {. }
$$

Определение 4. Весом wt $(x)$ набора $x$ из $F_{2}^{n}$ называется число единиц в $x$.

Определение 5. Расстояние между булевыми функциями $f_{1}$ и $f_{2}$ определяется как

$$
d\left(f_{1}, f_{2}\right)=\left|\left\{x \in F_{2}^{n} \mid f_{1}(x) \neq f_{2}(x)\right\}\right| .
$$

Определение 6. Нелинейность булевой функции $f$ над $F_{2}^{n}$ определяется как

$$
\operatorname{nl}(f)=\min _{l, \operatorname{deg}(l) \leqslant 1} d(f, l) .
$$

Определение 7. Для любого набора $u \in F_{2}^{n}$ значение

$$
W_{f}(u)=\sum_{x \in F_{2}^{n}}(-1)^{f(x)+\langle u, x\rangle}
$$

называется коэффициентом Уолша.

Нелинейность выражается через коэффициенты Уолша следующей формулой:

$$
\operatorname{nl}(f)=2^{n-1}-\frac{1}{2} \max _{u \in F_{2}^{n}}\left|W_{f}(u)\right|
$$

$\mathrm{B}$ [2] было доказано, что если $\operatorname{nl}(f)<\sum_{i=0}^{d}\left(\begin{array}{c}n \\ i\end{array}\right)$, то $\mathrm{AI}(f) \leqslant d+1$. Это эквивалентно нижней оценке нелинейности

$$
\mathrm{nl}(f) \geqslant \sum_{i=0}^{\mathrm{AI}(f)-2}\left(\begin{array}{l}
n \\
i
\end{array}\right) .
$$

Определение 8. Булева функция $f\left(x_{1}, \ldots, x_{n}\right)$ называется самодвойственной, если

$$
f\left(x_{1}+1, x_{2}+1, \ldots, x_{n}+1\right)=f\left(x_{1}, \ldots, x_{n}\right)+1 .
$$

Можно заметить, что если $f$ самодвойственная, то из того, что $f$ не имеет ненулевого аннигилятора степени меньше $k$, следует, что $f+1$ тоже не имеет ненулевого аннигилятора степени, меньшей $k$. Поэтому минимальные степени ненулевых аннигиляторов функщий $f$ и $f+1$ совпадают. Таким образом, для нахождения алгебраической иммунности самодвойственной функции $f$ достаточно ограничиться только аннигиляторами функщии $f$.

Лемма 1. Любой аннигилятор $g\left(x_{1}, \ldots, x_{n}\right)$ функции $l\left(x_{1}, \ldots, x_{n}\right), \operatorname{deg}(l)=1$, может быть представлен в виде

$$
g\left(x_{1}, \ldots, x_{n}\right)=f\left(x_{1}, \ldots, x_{n}\right)\left(l\left(x_{1}, \ldots, x_{n}\right)+1\right),
$$

гde

$$
\operatorname{deg}(f)=\operatorname{deg}(g)-1
$$


Доказательство. В силу аффинной эквивалентности можно считать, что $l=x_{1}+1$.

Рассмотрим запись $g\left(x_{1}, \ldots, x_{n}\right)$ в виде полинома. В силу того, что аннигиляторы функции образуют линейное пространство, после вычеркивания всех мономов, содержащих $x_{1}$, должна остаться функция $g_{1}\left(x_{2}, \ldots, x_{n}\right)$, такая, что $g_{1} l=g_{1}\left(x_{1}+1\right)=0$. Так как $g_{1}$ не зависит от $x_{1}$, то $g_{1}=0$. Следовательно, любой моном $g$ содержит $x_{1}$, тогда

$$
g\left(x_{1}, \ldots, x_{n}\right)=x_{1} f\left(x_{1}, \ldots, x_{n}\right)=(l+1) f
$$

где $\operatorname{deg}(f)=\operatorname{deg}(g)-1$.

Лемма 2. Пусть $l\left(x_{1}, \ldots, x_{n}\right)-$ булева функиия, $\operatorname{deg}(l)=1$. Тогда аннигиляторы функчии l степени, не большей $t$, образуют линейное пространство размерности $\sum_{i=0}^{t-1}\left(\begin{array}{c}n-1 \\ i\end{array}\right)$.

Доказательство. В силу аффинной эквивалентности можно считать, что $l=x_{1}+1$.

Рассмотрим произвольный аннигилятор $g\left(x_{1}, \ldots, x_{n}\right)$ функции $l\left(x_{1}, \ldots, x_{n}\right)$ такой, что $\operatorname{deg}(g) \leqslant t$. Рассмотрим запись $g\left(x_{1}, \ldots, x_{n}\right)$ в виде полинома. В силу того, что аннигиляторы функции образуют линейное пространство, после вычеркивания всех мономов содержащих $x_{1}$, должна остаться функщия $g_{1}\left(x_{2}, \ldots, x_{n}\right)$ такая, что $g_{1} l=g_{1}\left(x_{1}+1\right)=0$. Так как $g_{1}$ не зависит от $x_{1}$, то $g_{1}=0$. Следовательно, любой моном $g$ содержит $x_{1}$, тогда

$$
g\left(x_{1}, \ldots, x_{n}\right)=x_{1} f\left(x_{2}, \ldots, x_{n}\right)
$$

где $\operatorname{deg}(f) \leqslant t-1$.

При этом любая функщия $g\left(x_{1}, \ldots, x_{n}\right)=x_{1} f\left(x_{2}, \ldots, x_{n}\right)$, где $f\left(x_{2}, \ldots, x_{n}\right)$ - произвольная булева функция от $n-1$ переменной степени не выше $t-1$, является аннигилятором $l$ степени не выше $t$. Из этого следует утверждение леммы.

Доказательство следуюшего утверждения можно найти в [4], но мы решили привести его в нашей работе ввиду его простоты.

Лемма 3. Если $f-$ булева функция над $F_{2}^{n} u \mathrm{AI}(f)>k$, то

$$
\sum_{i=0}^{k}\left(\begin{array}{l}
n \\
i
\end{array}\right) \leqslant \operatorname{wt}(f) \leqslant \sum_{i=0}^{n-k-1}\left(\begin{array}{l}
n \\
i
\end{array}\right)
$$

Доказательство. Ищем аннигилятор функции $f$ методом неопределенных коэффициентов:

$$
g=a_{0}+\sum_{i=1}^{n} a_{i} x_{i}+\sum_{1 \leqslant i<j \leqslant n} a_{i j} x_{i} x_{j}+\ldots+\sum_{1 \leqslant i_{1} \leqslant \ldots \leqslant i_{k} \leqslant n} a_{i_{1} \ldots i_{k}} x_{i_{1}} \ldots x_{i_{k}}, \quad \operatorname{deg}(g) \leqslant k .
$$

Функщия $g$ является аннигилятором $f$ тогда и только тогда, когда $f(x)=1$ влечет $g(x)=0$. Тогда, для того чтобы $\mathrm{AI}(f)>k$, необходимо, чтобы получившаяся однородная система линейных уравнений на $a_{0}, a_{1}, a_{2}, \ldots$ имела бы только нулевое решение. Для этого необходимо, чтобы число неизвестных не превышало числа уравнений. Число уравнений равно $\operatorname{wt}(f)$, а число неизвестных $\sum_{i=0}^{k}\left(\begin{array}{l}n \\ i\end{array}\right)$. Следовательно, левое неравенство доказано. Применяя такие же рассуждения к $f+1$, получаем правое неравенство. 
Теорема 1. Пусть $f\left(x_{1}, \ldots, x_{n}\right)$ - булева функция над $F_{2}^{n}$ и $\mathrm{AI}(f)=k$, тогда

$$
\mathrm{nl}(f) \geqslant 2^{n-1}-\sum_{i=k-1}^{n-k}\left(\begin{array}{c}
n-1 \\
i
\end{array}\right)=2 \sum_{i=0}^{k-2}\left(\begin{array}{c}
n-1 \\
i
\end{array}\right)
$$

Доказательство. Представим нелинейность функции $f$ в виде

$$
\operatorname{nl}(f)=2^{n-1}-\frac{\alpha}{2}, \quad \alpha=\max _{u \in F_{2}^{n}}\left|W_{f}(u)\right| .
$$

Если $\max _{u \in F_{2}^{n}}\left|W_{f}(u)\right|$ достигается на нулевом наборе, тогда или $f$, или $f+1$ имеет вес $\left(2^{n}-\alpha\right) / 2$. Тогда в соответствии с леммой 3

$$
\frac{2^{n}-\alpha}{2} \geqslant \sum_{i=0}^{k-1}\left(\begin{array}{l}
n \\
i
\end{array}\right)
$$

Следовательно,

$$
\alpha \leqslant \sum_{i=k}^{n-k}\left(\begin{array}{l}
n \\
i
\end{array}\right) \leqslant 2 \sum_{i=k-1}^{n-k}\left(\begin{array}{c}
n-1 \\
i
\end{array}\right) \text {. }
$$

Отсюда получаем соответствующую оценку для нелинейности.

Если $\max _{u \in F_{2}^{n}}\left|W_{f}(u)\right|$ не достигается на нулевом наборе, тогда существует функщия $l\left(x_{1}, \ldots, x_{n}\right), \operatorname{deg}(l)=1$, такая, что

$$
d(f, l)=\frac{2^{n}-\alpha}{2}
$$

Функции $f$ и $l$ совпадают на $\left(2^{n}+\alpha\right) / 2$ наборах.

Заметим, что

$$
2^{n-1}-\alpha / 2=d(f, l)=\operatorname{wt}((f+1) l)+\mathrm{wt}(f(l+1)) .
$$

Отсюда

$$
\min \{\operatorname{wt}((f+1) l), \operatorname{wt}(f(l+1))\} \leqslant(1 / 2)\left(2^{n-1}-\alpha / 2\right)=2^{n-2}-\alpha / 4
$$

Если $\operatorname{wt}(f(l+1))<\operatorname{wt}((f+1) l)$, то определим $f_{1}=f, l_{1}=l+1$, в противном случае определим $f_{1}=f+1, l_{1}=l$.

Введем функцию $f_{2}=f_{1} l_{1}$. Тогда $\operatorname{wt}\left(f_{2}\right) \leqslant 2^{n-2}-\alpha / 4$.

Ищем аннигиляторы $g$ функщии $f_{2}$ степени не выше $k-2$ методом неопределенных коэффициентов:

$$
g=a_{0}+\sum_{i=1}^{n} a_{i} x_{i}+\sum_{1 \leqslant i<j \leqslant n} a_{i j} x_{i} x_{j}+\ldots+\sum_{1 \leqslant i_{1} \leqslant \ldots \leqslant i_{k-2} \leqslant n} a_{i_{1} \ldots i_{k-2}} x_{i_{1}} \ldots x_{i_{k-2}} .
$$

Функция $g$ является аннигилятором $f$ тогда и только тогда, когда $f(x)=1$ влечет $g(x)=0$. 
Следовательно, получаем однородную систему из не более чем $2^{n-2}-\alpha / 4$ линейных уравнений на $\sum_{i=0}^{k-2}\left(\begin{array}{c}n \\ i\end{array}\right)$ неизвестных. Пространство решений системы имеет размерность не менее $\sum_{i=0}^{k-2}\left(\begin{array}{c}n \\ i\end{array}\right)-\left(2^{n-2}-\alpha / 4\right)$.

В соответствии с леммой 2 , размерность пространства аннигиляторов функции $l_{1}$ степени не выше $k-2$ равна $\sum_{i=0}^{k-3}\left(\begin{array}{c}n-1 \\ i\end{array}\right)$.

Если

$$
\sum_{i=0}^{k-2}\left(\begin{array}{l}
n \\
i
\end{array}\right)-\left(2^{n-2}-\frac{\alpha}{4}\right)>\sum_{i=0}^{k-3}\left(\begin{array}{c}
n-1 \\
i
\end{array}\right)
$$

то найдется функция $f_{3}, \operatorname{deg}\left(f_{3}\right) \leqslant k-2$, такая что $f_{2} f_{3}=0$, но $f_{3} l_{1} \neq 0$, тогда $f_{3} l_{1}$ будет аннигилятором $f_{1}$, при этом $\operatorname{deg}\left(f_{3} l_{1}\right) \leqslant k-1$, что противоречит соотношению $\mathrm{AI}(f)=k$. Отсюда

$$
\begin{gathered}
\sum_{i=0}^{k-2}\left(\begin{array}{l}
n \\
i
\end{array}\right)-\left(2^{n-2}-\frac{\alpha}{4}\right) \leqslant \sum_{i=0}^{k-3}\left(\begin{array}{c}
n-1 \\
i
\end{array}\right), \\
\frac{\alpha}{4} \leqslant 2^{n-2}-\frac{1}{2} \sum_{i=k-2}^{n-k+1}\left(\begin{array}{c}
n-1 \\
i
\end{array}\right)+2^{n-2}-\left(2^{n-1}-\frac{1}{2} \sum_{i=k-1}^{n-k+1}\left(\begin{array}{c}
n \\
i
\end{array}\right)\right), \\
\frac{\alpha}{4} \leqslant \frac{1}{2}\left(\sum_{i=k-1}^{n-k+1}\left(\left(\begin{array}{c}
n-1 \\
i
\end{array}\right)+\left(\begin{array}{c}
n-1 \\
i-1
\end{array}\right)\right)-\sum_{i=k-2}^{n-k+1}\left(\begin{array}{c}
n-1 \\
i
\end{array}\right)\right)=\frac{1}{2} \sum_{i=k-1}^{n-k}\left(\begin{array}{c}
n-1 \\
i
\end{array}\right) .
\end{gathered}
$$

Следовательно,

$$
\mathrm{nl}(f) \geqslant 2^{n-1}-\sum_{i=k-1}^{n-k}\left(\begin{array}{c}
n-1 \\
i
\end{array}\right) .
$$

Следствие 1. Если п нечетно и $\mathrm{AI}\left(f\left(x_{1}, \ldots, x_{n}\right)\right)=\lceil n / 2\rceil$, то

$$
\mathrm{nl}(f) \geqslant 2^{n-1}-\left(\begin{array}{c}
n-1 \\
(n-1) / 2
\end{array}\right) \text {. }
$$

Заметим, что в [4] была построена функция от нечетного числа переменных $\boldsymbol{n}$ с алгебраической иммунностью $\lceil n / 2\rceil$ и нелинейностью $\operatorname{nl}(f)=2^{n-1}-\left(\begin{array}{c}n-1 \\ (n-1) / 2\end{array}\right)$. Из следствия 1 видно, что эта функция достигает границы (2), т.е. среди всех функций с максимально возможной алгебраической иммунностью она является функцией с наихудшей возможной нелинейностью. Подсчет ее нелинейности в [4] довольно труден и занимает много места. Теперь же нижняя оценка нелинейности функции из [4] сразу следует из следствия 1. Верхняя же оценка нелинейности функции из [4] будет следовать из теоремы 2, потому что эта функция является частным случаем функций $f_{n, k}$, возникающих в доказательстве теоремы 2. Заметим также, что в [3] для построенной там функции $f$ с нечетным числом переменных $n$ и алгебраической иммунностью $\lceil n / 2\rceil$ доказана нижняя оценка нелинейности $\mathrm{nl}(f) \geqslant 2^{n-1}-\left(\begin{array}{c}n-1 \\ (n-1) / 2\end{array}\right)$, которая, таким образом, совпадает с оценкой из следствия 1 для всех функций с таким числом переменных и такой алгебраической иммунностью. 
Следствие 2. Если $n$ четно и

$$
\operatorname{AI}\left(f\left(x_{1}, \ldots, x_{n}\right)\right)=\left\lceil\frac{n}{2}\right\rceil,
$$

mo

$$
\operatorname{nl}(f) \geqslant 2^{n-1}-\left(\begin{array}{c}
n \\
n / 2
\end{array}\right) .
$$

Заметим, что в [4] оценка следствия 2 была доказана для очень узкого класса функций.

Теорема 2. Оченка (1) неулучшаема для любого $n$ и любого $k \leqslant\lceil n / 2\rceil$. Более того, при любых допустимых параметрах существует уравновешенная функция, достигающая этой границы.

Доказательство. Покажем, что оценка (1) из теоремы 1 неулучшаема, предъявив для любого $n$ и любого $k \leqslant\lceil n / 2\rceil$ функцию $f\left(x_{1}, \ldots, x_{n}\right)$ такую, что

$$
\begin{aligned}
& \mathrm{AI}(f)=k, \\
& \mathrm{nl}(f)=2^{n-1}-\sum_{i=k-1}^{n-k}\left(\begin{array}{c}
n-1 \\
i
\end{array}\right) .
\end{aligned}
$$

Определим функщию $f_{n, k}$ следующим образом:

$$
f_{n, k}\left(x_{1}, \ldots, x_{n}\right)= \begin{cases}0, & \text { если } \operatorname{wt}\left(x_{1}, \ldots, x_{n}\right)<k, \\ 1, & \text { если } \operatorname{wt}\left(x_{1}, \ldots, x_{n}\right)>n-k, \\ x_{1}, & \text { если } k \leqslant \operatorname{wt}\left(x_{1}, \ldots, x_{n}\right) \leqslant n-k .\end{cases}
$$

Теперь докажем, что для любого $n$ и любого $k \leqslant\lceil n / 2\rceil$

$$
\begin{aligned}
& \mathrm{AI}\left(f_{n, k}\right)=k, \\
& \mathrm{nl}\left(f_{n, k}\right)=2^{n-1}-\sum_{i=k-1}^{n-k}\left(\begin{array}{c}
n-1 \\
i
\end{array}\right) .
\end{aligned}
$$

Несложно заметить, что

$$
f_{n, k}\left(x_{1}+1, x_{2}+1, \ldots, x_{n}+1\right)=f_{n, k}\left(x_{1}, \ldots, x_{n}\right)+1,
$$

т. е. $f_{n, k}$ - самодвойственная. Следовательно, функция $f_{n, k}$ является уравновешенной функцией.

В силу самодвойственности $f_{n, k}$, чтобы доказать , что $\mathrm{AI}(f) \geqslant k$, достаточно доказать, что $f_{n, k}+1$ не имеет ненулевого аннигилятора степени, меньшей $k$.

Запишем возможный аннигилятор функции $f+1$ степени не выше $k-1$ с помощью неопределенных коэффициентов:

$$
g=a_{0}+\sum_{i=1}^{n} a_{i} x_{i}+\sum_{1 \leqslant i<j \leqslant n} a_{i j} x_{i} x_{j}+\ldots+\sum_{1 \leqslant i_{1} \leqslant \ldots \leqslant i_{k-1} \leqslant n} a_{i_{1} \ldots i_{k-1}} x_{i_{1}} \ldots x_{i_{k-1}} .
$$

Функция $g$ является аннигилятором $f_{n, k}+1$ тогда и только тогда, когда $f(x)+1=1$ влечет $g(x)=0$. Получаем систему линейных уравнений на коэффициенты функщии $g$ :

$$
g\left(x_{1}, \ldots, x_{n}\right)=0
$$


для всех наборов $x$ таких, что $\operatorname{wt}(x) \leqslant k-1$.

Так как $g(0, \ldots, 0)=0$, то $a_{0}=0$. Так как $g(x)=0$, если wt $(x)=1$, то получаем, что $a_{i}=a_{0}=0$. Используя индукцию по весу наборов, получаем, что все коэффициенты $g$ равны нулю, следовательно, $g \equiv 0$. Получаем, что

$$
\mathrm{AI}\left(f_{n, k}\right) \geqslant k \text {. }
$$

Вычислим коэффициент Уолша функции $f_{n, k}$, соответствующий набору $(1,0, \ldots, 0)$, используя самодвойственность $f_{n, k}$ :

$$
\begin{aligned}
W_{f_{n, k}}(1,0, \ldots, 0) & =\sum_{\left(x_{1}, \ldots, x_{n}\right) \in F_{2}^{n}}(-1)^{f_{n, k}\left(x_{1}, \ldots, x_{n}\right)+x_{1}} \\
& =2^{n}-2 \operatorname{wt}\left(f_{n, k}\left(x_{1}, \ldots, x_{n}\right)+x_{1}\right) \\
& =2^{n}-2\left(\operatorname{wt}\left(f_{n, k}\left(0, x_{2}, \ldots, x_{n}\right)\right)+\operatorname{wt}\left(f_{n, k}\left(1, x_{2} \ldots, x_{n}\right)+1\right)\right) \\
& =2^{n}-4 \operatorname{wt}\left(f_{n, k}\left(0, x_{2}, \ldots, x_{n}\right)\right) \\
& =2^{n}-4 \sum_{i=n-k+1}^{n-1}\left(\begin{array}{c}
n-1 \\
i
\end{array}\right) \\
& =2 \sum_{i=k-1}^{n-k}\left(\begin{array}{c}
n-1 \\
i
\end{array}\right) .
\end{aligned}
$$

Следовательно,

$$
\mathrm{nl}\left(f_{n, k}\right) \leqslant 2^{n-1}-\sum_{i=k-1}^{n-k}\left(\begin{array}{c}
n-1 \\
i
\end{array}\right) .
$$

Тогда из теоремы 1 следует, что

$$
\operatorname{AI}\left(f_{n, k}\right) \leqslant k
$$

поэтому

$$
\mathrm{AI}\left(f_{n, k}\right)=k
$$

следовательно,

$$
\mathrm{nl}\left(f_{n, k}\right) \geqslant 2^{n-1}-\sum_{i=k-1}^{n-k}\left(\begin{array}{c}
n-1 \\
i
\end{array}\right)
$$

отсюда

$$
\mathrm{nl}\left(f_{n, k}\right)=2^{n-1}-\sum_{i=k-1}^{n-k}\left(\begin{array}{c}
n-1 \\
i
\end{array}\right) .
$$

Автор выражает глубокую благодарность Ю. В. Таранникову за постановку задачи, внимание к работе и ценные советы. 


\section{Список литературы}

1. Courtois N., Meier W., Algebraic attacks on stream ciphers with linear feedback. Lecture Notes Computer Sci. (2003) 2656, 345-359.

2. Dalai D. K., Gupta K. C., Maitra S., Results on algebraic immunity for cryptographically significant Boolean functions. Lecture Notes Computer Sci. (2004) 3348, 92-106.

3. Dalai D. K., Gupta K. C., Maitra S., Cryptographically significant Boolean functions: construction and analysis in terms of algebraic immunity. Lecture Notes Computer Sci. (2005) 3557, 98-111.

4. Dalai D. K., Maitra S., Sarkar S., Basic theory in construction of Boolean functions with maximum possible annihilator immunity. Design, Codes and Cryptography (2006) 40, 41-58.

5. Meier W., Pasalic E., Carlet C., Algebraic attacks and decomposition of Boolean functions. Lecture Notes Computer Sci. (2004) 3027, 474-491.

Статья поступила 20.07.2006. 